${ }^{\circledR}$ Entomologica Fennica. 19.X.1992

\title{
Spiders (Araneae) from Central Yakutia, Siberia
}

\author{
Seppo Koponen \& Yuri M. Marusilk
}

Koponen, S. \& Marusik, Yu. M. 1992: Spiders (Araneae) from Central Yakutia, Siberia. — Entomol. Fennica 3:163-166.

A spider collection from the central parts of Yakutia, Siberia, includes 183 species, of which 130 have not been previously recorded from Yakutia. The species are listed together with collecting localities and with the first published record from Yakutia.

Seppo Koponen, Zoological Museum, University of Turku, SF-20500 Turku, Finland

Yuri M. Marusik, Institute for Biological Problems of the North, Russian Academy of Sciences, K. Marx pr. 24, Magadan 685010, Russia

\section{Introduction}

Yakutia, comprising the northeastern part of Central Siberia, consists of an area of about $3100000 \mathrm{~km}^{2}$. Yakutia borders in the south on the Amur region and in the north on the Arctic Ocean. Very little up to now has been known about the spider fauna of this huge area. The paper by Kulczynski (1908), who recorded 60 species (26 of the present species) from Yakutia, can be mentioned. In addition, some data have been published, especially by Russian authors, in recent years.

The senior author collected spiders in July 1977 in the central parts of Yakutia. The study area lies mainly in the taiga zone, dominated by larch forests. In addition to forests, steppes, meadows, pastures, bogs, shore habitats as well as villages were included in the collecting habitats.

\section{Study area, material and methods}

The collecting areas can be divided into three groups: localities near Yakutsk (no. 1-3, 7), Lyampeshka River (4) and sites on the Vilyuy
River (5-6), see Fig. 1. Material was collected using sweep-netting, sieving moss and litter, and hand-picking. The study sites were:

1) Oktemtsy, about $50 \mathrm{~km} \mathrm{SW}$ of Yakutsk in the valley of the Lena River, about $61^{\circ} 30^{\prime} \mathrm{N}$, $129^{\circ} 30^{\prime} \mathrm{E}$ : river-side steppes, pastures, Salix viminalis bushes, slopes between steppe and taiga, marginal parts of Larix-dominated taiga forest with meadows and "alas"-ponds. Collecting period: 7-10 \& 13 July 1977.

2) Ulakhan-An, about $100 \mathrm{~km} \mathrm{SW}$ of Yakutsk: Larix taiga, river valley with meadows; 11 July 1977.

3) $20 \mathrm{~km} \mathrm{~S}$ of Bulgunyakhtakh $(80 \mathrm{~km} \mathrm{~S}$ of Oktemtsy), the Lena River valley: cambric cliffs, river-shore stone beds, taiga edge; 12 July 1977.

4) Lyampeshka (Lepiske) River joining the Lena River, about $64^{\circ} 40^{\prime} \mathrm{N}, 125^{\circ} 30^{\prime} \mathrm{E}$ : river-side meadows with Salix viminalis, taiga (Larix, Picea, Pinus, also P. pumila, and Betula), pine bogs, swamps, "alas"-ponds; 15-20 July 1977.

5) El'gyay, in the valley of the Vilyuy River, about $62^{\circ} 20^{\prime} \mathrm{N}, 117^{\circ} 40^{\prime} \mathrm{E}$ : meadows, "alas"ponds, taiga; 26-28 July 1977. 


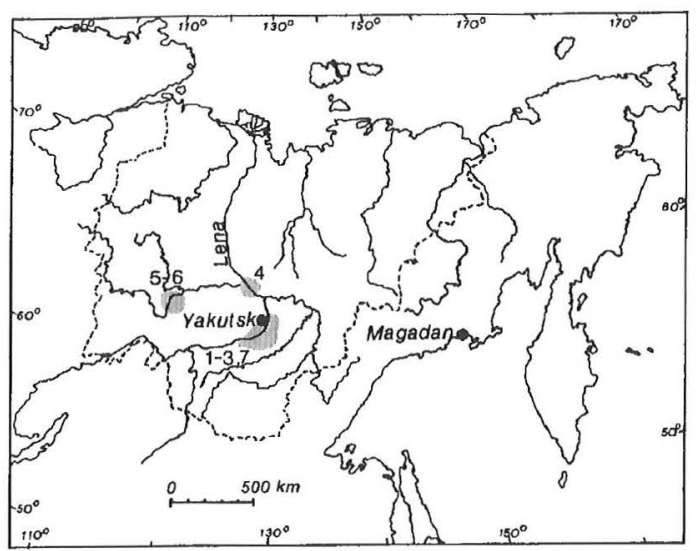

Fig. 1. Location of the study areas (shaded) in Yakutia (border indicated by broken line). The numbers refer to the collecting sites (see Study area, material and methods).

7) $20-40 \mathrm{~km} \mathrm{~N}$ of Yakutsk: moist meadow, dry meadow and "alas"-pond, fen, peat-bog; 30 July 1977.

The material is mainly deposited in the Zoological Museum, University of Turku.

\section{Spider fauna of Central Yakutia}

The spiders found are listed in Table 1 . The total number of species is 183, of which 130 are new to Yakutia. Linyphiidae (s. lat.) dominated species numbers (67 species or $37 \%$ of total). Other species-rich families were Lycosidae (17 species), Araneidae (15), Salticidae (15), Philodromidae (14), Gnaphosidae (12) and Theridiidae (11).

A few species hitherto known only from the West Palaearcticum, like Trichonchus hackmani, Sitticus distinguendus and Robertus scoticus, are new to the fauna of Siberia. Many of the present species have previously been found in Siberia only in the Magadan area (Marusik \& Eskov unpubl.). About half of the species found in Yakutia are known also from Fennoscandia.

Four species were collected at six of the total of seven sites and eight species at five sites; as many as 101 species were found at one site only. The special character of site 4 (Lyampeshka
River) is indicated by the great number (41) of species found only there compared to site 1 (Oktemtsy: 21).

Acknowledgements. The generous help given to S. Koponen by the late Dr. Yuri N. Ammosov and by Dr. Nikolay N. Vinokurov, as well as by the entire staff of the Entomological Laboratory of the Yakutian Academy of Sciences during the field work in 1977 is gratefully acknowledged. The trip was a part of the scientific exchange programme of the Academy of Finland and the Academy of Sciences of the USSR. Yu. M. Marusik thanks Dr. Pekka T. Lehtinen (Turku) for help in organizing a trip to Finland. Drs. Michael I. Saaristo (Turku), Dmitry V. Logunov (Novosibirsk), Kirill Yu. Eskov, Kirill G. Mikhailov and Andrej V. Tanasevitch (Moscow), and Alexey A. Zyuzin (Alma-Ata) provided valuable information. Ms. Heli Hurme drew the figure.

\section{References}

Eskov, K. Yu. (Еськов, К. Ю.) 1985: The spiders of tundra-zone in the USSR. (In Russian) - Proc. Zool. Inst., Leningrad 139:121-129.

- 1987: The spider genus Robertus O. Pickard-Cambridge in the USSR, with an analysis of its distribution (Arachnida: Araneae: Theridiidae). - Senckenbergiana Biol. 67:279-296.

- 1988: Aranei in Central Siberia. — In: Rogachova, E. V. (Рогачова, Е. В.) (ed.), Materials on the fauna of Central Siberia and adjacent regions of Mongolia. (In Russian): 101-155. Moscow.

Eskov, K. Yu. \& Marusik, Yu. M. 1991: New linyphiid spiders (Araneae, Linyphiidae) from East Siberia. Korean Arachnol. 6:237-253.

Hippa, H. \& Oksala, I. 1985: A review of some Holarctic Agyneta Hull s.str. (Araneae, Linyphiidae). - Bull. Br. Arachnol. Soc. 6:277-288.

Koponen, S., Caselius, R. \& Itämies, J. 1990: Notes on the wolf spider Pardosa plumipes (Araneae, Lycosidae). - Memoranda Soc. Fauna Flora Fennica 66:135-136.

Kronestedt, T. 1986: Studies on species of Holarctic Pardosa groups (Araneae, Lycosidae). III. Redescriptions of Pardosa algens (Kulczynski), P. septentrionalis (Westring), and P. sodalis Holm. - Entomol. Scand. 17:215-234.

Kulczynski, W. 1908: Araneae et Oribatidae expeditionum rossicarum in insulas Novo-Sibiricas annis 1885-1886 et 1900-1903 susceptarum. - Mem. Acad. Imper. Sci. St.-Petersbourg 18(7):1-97.

Marusik, Yu. М. (Марусик, Ю. М.) 1991: Crab spiders of the family Philodromidae (Aranei) from East Siberia. (In Russian with English summary) - Zool. Zh. 70(10):48-58.

Mikhailov, K. G. 1990: The spider genus Clubiona Latreille, 1804 in the Soviet Far East, 1 (Arachnida, Aranei, Clubionidae). - Korean Arachnol. 5:139-175. 
- 1991: The spider genus Clubiona Latreille 1804 in the Soviet Far East, 2 (Arachnida, Aranei, Clubionidae). - Korean Arachnol. 6:207-235.

Ovtsharenko, V. I. \& Marusik, Yu. М. (Овчаренко, В. И. \& Марусик, Ю. М.) 1988: Spiders of the family Gnaphosidae (Aranei) of the North-East of the USSR (The Magadan Province). (In Russian) - Entomol. Obozr. 67:204-217.
Proszynski, J. 1979: Systematic studies on East Palaearctic Salticidae, III. Remarks on Salticidae of the USSR. Ann. Zool. 21(11):299-369.

Utotchkin, A. S. (У точкин, А. С.) 1968: [Spiders of the genus Xysticus of the USSR fauna.] (In Russian) Perm University. 73 pp.

Received 2.IV.1992

Table 1. List of species collected from Yakutia in 1977. The sites and the first published record from Yakutia are also given: * = this report, O.\&M.'88 = Ovtsharenko \& Marusik 1988, H.\&O.'85 = Hippa \& Oksala 1985.

\begin{tabular}{|c|c|c|c|c|c|}
\hline Family/species & Sites & First record & Family/species & Sites & First record \\
\hline \multicolumn{3}{|l|}{ Amaurobiidae (2) } & G. proxima Kulczynski & 4 & Kulczynski'08 \\
\hline Arctobius agelenoides (Emerton) & 4,6 & * & G. sticta Kulczynski & 4 & Kulczynski'08 \\
\hline Titanoeca nivalis Simon & 4 & * & Haplodrassus hiemalis (Emerton) & 4 & \\
\hline \multicolumn{3}{|l|}{ Araneidae (15) } & H. moderatus (Kulczynski) (?) & 4 & \\
\hline Aculepeira packardi (Thorell) & & & Micaria lenzi Bösenberg & 1,3 & \\
\hline Araneus alsine (Walckenaer) & $\begin{array}{r}1,2,5,6,1 \\
2,3\end{array}$ & $\begin{array}{r}\text { Kulczynskio8 } \\
*\end{array}$ & M. rossica Thorell & $1,4,5$ & \\
\hline A. marmoreus (Clerck) & $\begin{array}{r}2,3 \\
1,3,4,5\end{array}$ & * & Zelotes potanini Schenkel & 3 & \\
\hline A. nordmanni (Clerck) & $1,2,4$ & * & Hahniidae (1) & & \\
\hline \multirow[t]{2}{*}{ A. yukon Levi } & $1,2,4,6$ & Kulczynski'08 & Hahnia ononidum Simon & 4 & \\
\hline & \multicolumn{2}{|c|}{ (as A. quadratus) } & & & \\
\hline \multirow{2}{*}{\multicolumn{6}{|c|}{$\begin{array}{l}\text { Linyphiidae (Linyphiinae) (20) } \\
\text { Agyneta olivacea (Emerton) }\end{array}$}} \\
\hline & 1,4 & * & \multirow{2}{*}{\multicolumn{3}{|c|}{ (present material) }} \\
\hline Cercidia prominens (Westring) & 4 & * & & & \\
\hline Cyclosa conica (Pallas) & $1,3,4$ & * & A. affinisoides Tanasevitch & 1 & \\
\hline C. oculata (Walckenaer) & 7 & * & Allomengea scopigera (Grube) & 1 & \\
\hline Cyphepeira silvicultrix (C. L. Koch & ch) & * & Bathyphantes setiger F.O.P.-Cambr & 6 & \\
\hline Hypsosinga albovittata (Westring) & g) & * & Estrandia grandaeva (Keyserling) & 4 & \\
\hline Larioides cornutus (Clerck) 1 & $1,3,4,5,6,7$ & Kulczynski’08 & Kaestneria anceps (Kulczynski) & 6 & \\
\hline L. patagiatus (Clerck) & $1,4,5,6$ & Kulczynski’08 & Lepthyphantes dybowskii (O.P.-Can & mbr.) 4 & \\
\hline \multirow[t]{2}{*}{ Singa hamata (Clerck) } & 3,4 & * & L. karpinskii (O.P.-Cambridge) & 4,6 & \\
\hline & & & L. kochiellus Strand & $1,4,6$ & \\
\hline Argyronetidae (1) & & & L. nebulosus (Sundevall) & 6 & \\
\hline Argyroneta aquatica (Clerck) & 1 & Kulczynski’08 & L. taczanowskii (O.P.-Cambridge) & 4,6 & \\
\hline Clubionidae (5) & & & L. unicornis (O.P.-Cambridge) & 1,6 & \\
\hline Cheiracanthium cf. erraticum & $1,5,6,7$ & * & Macra & & \\
\hline Clubiona interjecta L. Koch & $1,4,5,6$ & Kulczynski'08 & (O.P.-Cambridge) & 4 & \\
\hline C. kulczynskii de Lessert & $3,4,6$ & Mikhailov'91 & Microlinyphia impigra (O.P.-Cambri & idge) 5 & \\
\hline C. latericia Kulczynski & 4,5 & Mikhailov'90 & M. pusilla (Sundevall) & $2,4,6,7$ & \\
\hline C. riparia L. Koch & 1,4 & * & Oreonetides helsdingeni Eskov & 4 & \\
\hline & & & O. vaginatus (Thorell) & 1 & \\
\hline $\begin{array}{l}\text { Dictynidae (7) } \\
\text { Argenna prominula Tullaren }\end{array}$ & & & Pityohyphantes phrygianus & & \\
\hline Argenna prominula Tullgren & 6 & * & (C. L. Koch) & 4,6 & \\
\hline Dictyna alaskae Chamberlin \& IV & vie & * & Poeciloneta tanasevitchi Marusik & 4 & \\
\hline D. arundinacea (Linnaeus) & 2,7 & * & Porrhomma borealis (Banks) & 2 & \\
\hline D. major Menge & 4 & $\begin{array}{r}\text { Kulczynski'08 } \\
\text { (as D. sibirica) }\end{array}$ & Linyphiidae (Erigoninae) (47) & & \\
\hline D. pusilla Thorell & 4 & * & Carorita limnea (Crosby \& Bishop) & 4,7 & \\
\hline D. uncinata Thorell & 4 & * & Ceratinops sp. & 4 & \\
\hline Emblyna annulipes (Blackwall) & 4 & * & Diplocentria bidentata (Emerton) & 6 & \\
\hline Dolomedidae (1) & & & Diploceph & & \\
\hline Dolomedes buhkaloi Marusik & & & & 6 & \\
\hline & $1,4,5,6,7$ & Kulczynski'08 & D. uliginosus Eskov & 1,4 & \\
\hline & & & bifrons (Blackwall) & 4 & \\
\hline Gnaphosidae (12) & & & Enidia bituberculata (Wider) & $3,4,6$ & \\
\hline Drassodes neglectus (Keyserling & g) & O.\&M.'88 & Entelecara media Kulczynski & 4 & Eskov'88 \\
\hline D. pubescens (Thorell) & 5,6 & * & Erigone arcticola Chamberlin \& Ivie & 5 & \\
\hline Callilepis nocturna (Linnaeus) & 4 & * & E. atra Blackwall & $4,5,6,7$ & \\
\hline Gnaphosa nigerrima L. Koch & 5,6 & * & Glyphesis asiaticus Eskov & 7 & \\
\hline G. muscorum (L. Koch) & 1,5 & * & Gnathonarium dentatum (Wider) & 1,7 & \\
\hline
\end{tabular}




\begin{tabular}{|c|c|c|c|c|c|}
\hline Family/species & Sites & First record & Family/species & Sites & First record \\
\hline G. taczanowskii (O.P.-Cambridge) & 5 & * & P. aryy Marusik & 2 & Marusik'91 \\
\hline Hilaria devitata Eskov & 4 & * & P. aureolus (Clerck) & $1,2,4$ & \\
\hline \multirow{2}{*}{$\begin{array}{l}\text { H. herniosa (Thorell) } \\
\text { H. holmi Eskov }\end{array}$} & 1,6 & * & P. cespitum (Walckenaer) & 4 & Kulczynski'08 \\
\hline & 4 & * & & (as P.a & ureolus sibirica) \\
\hline $\begin{array}{l}\text { H. holmi Eskov } \\
\text { H. minuta Eskov }\end{array}$ & 4,6 & * & P. fuscomarginatus (de Geer) & 1 & Marusik'91 \\
\hline H. tatrica Kulczynski & 6 & * & P. histrio (Latreille) & 1 & Marusik'91 \\
\hline Hilaira sp. & 6 & * & P. mysticus Dondale \& Redner & 3 & Marusik'91 \\
\hline \multicolumn{2}{|c|}{ Hybauchenidium aquilonare (L. Koch) } & Eskov'85 & P. praedatus (O.P.-Cambridge) & $1,4,5,6$ & \\
\hline \multicolumn{2}{|c|}{ Hypselistes jacksoni (O.P.-Cambr.) 3,4,6 } & * & P. vinokurovi Marusik & 1,4 & Marusik'91 \\
\hline Lasiargus laricetorum Eskov & 6 & * & Thanatus albomaculatus Kulczynsk & 1 & Kulczynski'08 \\
\hline \multirow{2}{*}{$\begin{array}{l}\text { Maso sundevalli (Westring) } \\
\text { Minicia exarmata Eskov }\end{array}$} & 4 & * & T. mediocris Kulczynski & 4 & Kulczynski'08 \\
\hline & 3 & * & Tibellus asiaticus Kulczynski & 4,7 & Kulczynski'08 \\
\hline \multicolumn{2}{|c|}{ Minyrioloides trifrons (O.P.-Cambridge) } & * & T. maritimus (Menge) & 6 & Marusik'91 \\
\hline Notioscopus jamalensis Grese & 4,6 & * & Salticidae (15) & & \\
\hline $\begin{array}{l}\text { Oedothorax retusus (Westring) } \\
\text { Praestigia pini }(\mathrm{Holm})\end{array}$ & 5,6 & * & Bianor stepposus Logunov & 1,2 & \\
\hline $\begin{array}{l}\text { Praestigia pini (Holm) } \\
\text { Proceracumbium sibiricus Fskov }\end{array}$ & 2 & * & Dendryphantes fusconotatus (Grub & abe) $1,2,3,4$ & ,7 Proszynski'79 \\
\hline \multirow{2}{*}{\multicolumn{2}{|c|}{$\begin{array}{l}\text { Proceracymbium sibiricus Eskov } \\
\text { Savignya nenilini Marusik }\end{array}$}} & * & D. rudis (Sundevall) & 1 & Proszynski'79 \\
\hline & 1,7 & * & Evarcha arcuata (Clerck) & $1,2,4,7$ & Proszynski'79 \\
\hline Scotinotylus alienus (Kulczynski) & 7 & * & Heliophanus baicalensis Kulczynsk & $s k i \quad 1$ & \\
\hline $\begin{array}{l}\text { Scotinotylus sp. } \\
\text { Dactylopisthes video (Chamb. \& }\end{array}$ & Ivie) $\begin{array}{r}1 \\
2,4\end{array}$ & * & H. camtschadalicus Kulczynski & 4 & Proszynski'79 \\
\hline Silometopus sibiricus Eskov & (vie) $\begin{array}{r}2,4 \\
4,7\end{array}$ & * & H. lineiventris Simon & 1 & \\
\hline Tibioploides arcuatus (Tullgren) & 4 & * & H. patagiatus Thorell & 4 & * \\
\hline Tmeticus affinis (Blackwall) & 5 & * & Marpissa pomatia (Walckenaer) & 1,4 & Proszynski'79 \\
\hline T. tolli Kulczynski (?) & 7 & Kulczynski'08 & Pellenes ingnifrons (Grube) & 5,6 & Proszynski'79 \\
\hline Trichoncus hackmani Millidge & 2 & & Phlegra fuscipes Kulczynski & 1 & \\
\hline Tubercithorax furcifer Eskov & 4 & * & Sitticus cutleri Proszynski & 1,4 & * \\
\hline Veles wagae (O.P.-Cambridge) & 1 & * & S. distinguensis (Simon) & 3 & \\
\hline Walckenaeria clavicornis (Emerto & n) & * & S. finschi (L. Koch) & 5 & Kulczynski'08 \\
\hline W. fraudatrix Millidge & 4 & * & S. floricola (C. L. Koch) & $1,4,6$ & Proszynski'79 \\
\hline W. holmi Millidge & 4,7 & * & Tetragnathidae (4) & & \\
\hline W. lepida (Kulczynski) & 4 & * & Pachygnatha clercki Sundevall & 5,6 & Kulczynski'08 \\
\hline Zornella cultrigera (L. Koch) & 1 & * & Tetragnatha dearmata Thorell & $1,2,4,6$ & \\
\hline Erigoninae sp. 1 & 7 & & T. extensa (Linnaeus) & $2,3,4,5,6$ & Kulczynski'08 \\
\hline Erigoninae sp. 2 & 4 & & T. pinicola L. Koch & 1 & \\
\hline Lycosidae (17) & & & Theridiidae (11) & & \\
\hline Alopecosa albostriata (Grube) & 4 & Kulczynski'08 & us Eskov & 4,7 & Eskov'87 \\
\hline A. borea (Kulczynski) & 1 & Kulczynski'08 & Jackson & 6 & \\
\hline A. sibirica (Kulc & 3 & Kulczynski'08 & maculata (de Geer) & 1,2 & \\
\hline Pardosa adustella Roewer & $1,3,4,5,7$ & * & S. bipunctata (Linnaeus) & 6 & * \\
\hline P. andersoni Gertsch & 5,6 & * & Theridion impressum L. Koch 1,2, & $2,3,5,6,7$ & * \\
\hline P. atrate & $1,5,6,7$ & * & T. ohlerti Thorell & $1,4,5$ & * \\
\hline (Thorell) & 2,7 & * & Koch & 7 & * \\
\hline P. lascive & 6 & * & T. pictum (Walckenaer) & $1,2,4,5,6$ & * \\
\hline a (Odenwall) & & * & T. sibiricum Marusik & 3 & \\
\hline P. plumipes (Thorell) & $1,3,5,6$ & Koponen\&al'90 & T. varians Hahn & $1,4,6$ & * \\
\hline sert & $1,5,7$ & & Thymoites bellisimum (L. Koch) & 4 & \\
\hline & & Kronestedt'86 & Thor & & \\
\hline & & & Heriaeus mellottei $\mathrm{Si}$ & 1 & \\
\hline $\begin{array}{l}\text { P. tesquorum (Odenwall) } \\
\text { Pardosan. sp. }\end{array}$ & & Kulczynski’08 & Misumena vatia (Clerck) & $1,2,4,6,7$ & \\
\hline (will be $\mathrm{d}$ & lescribed & oy $A$. & Ozyptila sincera Kulczynski & $1,2,4$ & * \\
\hline Pirata piraticus (Clerck) & $1,5,6$ & & Tmarus rimosus Paik (?) & 1 & * \\
\hline P. pra & & * & Xysticus audax (Schrank) & 1,2 & * \\
\hline Xerolycosa nemoralis (Westring) & 6 & * & X. britcheri Gertsch & 1,4 & \\
\hline $\begin{array}{l}\text { Oxyopidae ( } \\
\text { Oxyopes cf. ral }\end{array}$ & 1247 & & $X$. emertoni Keyserling & 4,6 & $\begin{array}{r}\text { Kulczynski'08 } \\
\text { (as Xexcellans) }\end{array}$ \\
\hline & $1,2,4,1$ & mosus) & & 1 & \\
\hline & & & X. vachoni Schenkel & 1,7 & Utotchkin'68 \\
\hline Apollophanes lenensis Marusik & & Marusik'91 & & & (as X.jacuticus) \\
\hline Philodromus alascensis Keyserlin & & $\begin{array}{r}\text { Kulczynski'08 } \\
\text { (as P. varians) }\end{array}$ & $\begin{array}{l}\text { Zoridae (1) } \\
\text { Zora cf. nemoralis }\end{array}$ & 4 & \\
\hline
\end{tabular}

